Mario Doria

Trieste

\title{
SULLA STORIA DEL TOPONIMO ISTRIANO RABAC
}

Il nome della nota località balneare istriana Rabac (in grafia italianeggiante $R a$ $b a z)$ è attestato già nel 1341 sotto la forma Rabaç, precisamente negli Statuti di Albona [Labin] (cfr. P. Kandler "L'Istria" III, 1848, pp. 14 s.). A questo Rabaç fa riscontro, nel '500, Rabaz, che incontriamo nell'Itinerario Bragadin-Lando-Morosini dell'a. 1554 (ed. M. Bertoša VHARP 17, 1972, p. 41) e nel Catastico di Fabio da Canal dell'a. 1566 (ed. D. Klein, ib. 11-12, 1966-67, pp. 16 - bis, 62). Rabaz ricompare in Carlo Donadoni, a. 1719 (P. Kandler Emporio p. 96, in "Miscellanea Conti" 1861 -62), in un documento dell'a. 1749 (P. Kandler cit. p. 282), nonché nel Catasto di V. Morosini IV, a. 1775-76 (ed. M. Bratulić, Trieste-Fiume 1980, pp. 349-352). Anche la cartografia veneta di fine ' 700 attesta la forma Rabaz, così la nota carta dell'Istria Meridionale di Giov. Valle (Venezia 1784). Rabaz ricompare nel Reperto-Bargnani dell'a. 1806 (ed. E. Apih, ACRSR 12, 1981-82, p. 219), nell'"Avviso della Commissione per la vendita dei beni dello Stato del Litorale", Trieste 15-1-1825 (Archivio di Stato, per gentile informazione del dott. Pierpaolo Dorsi), in Carlo Combi a. 1858 -59 (cfr. E. Apih cit. p. 321) ecc. Rammenteremo anche la forma Rabatz (alternante con Rabaz) in R. P. Burton Note sopra i Castellieri (Capodistria 1877) p. 35 e Rabas (nella locuzione Porto Rabas in alcune carte geografiche del 1753 e 1780, Lago-Rossit DH indici), nonché in P. Tedeschi Viaggio fantastico in Oga Magoga, 1863, su cui v. P. Blasi "Voce Giul.“ 1-6-1984 p. 4). Abbastanza comune anche la locuzione Porto Rabaz, soprattutto nella cartografia istriana a partire dall'a. 1620 fino al 1797 (vedi gli indici in Lago-Rossit cit.): ricorderemo fra queste la Carta Geografica del Coronelli (Venezia 1696) nonché la Carta Santini ("à Venise" ante 1780, cfr. fot. in E. Schwarzenberg PIstr. 44 s. V, f. 8-9, 1980, p. 12); fra i moderni citeremo M. Gerbini Quaderni di Fianona (Trieste 1976) p. 41 e M. Catano, "In Strada Granda“ N. 27 (aprile 1986) p. 26 e qualche altro.

Com'è noto, durante il periodo della sovranità italiana in Istria, la località fu designata, normalmente, con la locuzione Porto Albona. L'avvio a questa forzata italianizzazione ${ }^{1}$ era stato dato dal glottologo albonese Matteo Bartoli (Riflessi slavi, 1908, p. 50), il quale proponeva per l'appunto, al posto di Rabac la dizione Porto d'Albona. A dir il vero la dicitura Porto d'Albona non ha avuto troppa fortuna (la ritrovo ripetuta, per quanto io ne sappia, solamente nel Portolano del Mediterraneo, f. V, Genova 1928, p. 178). Più comune, invece, la locuzione abbreviata Porto

\footnotetext{
Si noti, tuttavia, che Porto Albona riesce a filtrare anche nella stampa jugoslava moderna, ad es. in Guida Turistica d'Istria (Pula 1982) p. 42 (in alternanza con Rabac), ib. p. 30 "Posto (sic) Albona" (/Rabac).
} 
Albona" ${ }^{2}$ Secondo un redattore (M. V.) di "Voce Giuliana" (16-6-1979 p. 3) sarebbero stati gli abitanti stessi di Rabac a desiderare, nel 1920, che la località fosse chiamata Porto Albona (e difatti, a partire da quella data tale dicitura risulta, nella stampa italiana, ampiamente documentata, anche se non vengono poste del tutto in oblì le forme originarie Rabaz o Rabac). Sennonché c'è forse un po' da dubitare sul carattere "popolare" di tale iniziativa: più che dagli abitanti di Rabac essa sarà stata espressa dagli italiani di Albona, i quali, alla lor volta, potevano vantare un illustre precedente, l'autorità del conterraneo Matteo Bartoli. Ma c'è di più: a mio avviso si può fare un ulteriore passo in tema di "suggerimenti": poiché è assai verosimile che il Bartoli stesso "creò" il nuovo toponimo sulla base di un altro precedente, piuttosto significativo, il passo già citato del Burton, il quale definisce Rabatz (Rabaz) per l'appunto "il porto di Albona“. E per inciso aggiungerò che la medesima locuzione è presente anche in un'altra opera dello stesso autore, Il litorale istriano (rist.traduzione Trieste 1973) p. 109, in cui si dice testualmente (cito dalla traduzione) "Rabac, la marina ${ }^{3}$ e porto d'Albona“. Inconsapevolmente, dunque, sarebbe stato il Burton il responsabile della poco felice italianizzazione del toponimo.

Assodato, ora, che la località ebbe inequivocabilmente, fin dalle sue prime menzioni, un solo nome, quello di Rabac, sarà opportuno di fissarne, anche, l'etimo. Le proposte, a dire il vero, scarseggiano e si limitano ad un solo tentativo, il collegamento di Rabac col nome cr. dell'isola di Arbe, Rab. Così il Bartoli, o.c. p. 50. A sostegno potremmo addurre il fatto che Rabac, nel 1215, strinse un patto di pace e d'amicizia sul mare con l'isola di Arbe (cfr. C. Nider "Voce Giul." 1-1-1986). Ma ciò non basta: per poter rafforzare quest'ipotesi bisognerebbe, anche, accertare che prima di questa data la località aveva un altro nome (a parte il fatto che la "notizia" relativa a questo patto potrebbe essere stata, al limite, "costruita" sulla base nell'assonanza, casuale, di $R a b$ con $R a b a c$ ). In assenza di elementi probatori in favore di Rabac da Arbe, mi si permetta di suggerire un'altra spiegazione, forse più semplice e quindi più convincente. Tenuto anche conto di una testimonianza che ho voluto, deliberatamente, lasciare per ultima, precisamente il particolare che Rabac è anche il nome di un torrentello che sbocca nel porto omonimo (Portol. del Medit. cit. p. 134), il nostro toponimo (in origine un idronimo) potrebbe derivare da un appellativo croato rabac significante quello che in veneziano si dice servidor, canale di deflusso delle acque tipico degli stabilimenti saliniferi. Quest' impiego di rabac ha il suo parallelo nel cr. di Dalmazia, precisamente nei pressi di Zara, dove rabac significa, appunto, "sotto corbolo, servidor" ed è sinonimo anche di (veneto) dalm. abdello (da lat. BEDALE "fosso di acqua corrente"), così A. Zamboni in "Festschrift Muljačić“ (Hamburg 1987) p. 265. Inutile sottolineare, tanto è evidente, che il signi-

$2 \quad$ A questo proposito sarà forse utile precisare che questo marina d'Albona non ha nulla a che fare con la località Marina d'Albona, che, insieme a Porto Marina (a. 1556, 1780) e a Marina ("La Voce del Popolo" 2-11-1984, anche Marina o Ravni, ib. 18-10-1985 s. v.) non è altro che l'abbrevazione del più lungo Santa Marina (d'Albona) o Porto Santa Marina (per le cui attestazioni rimando al Dizionario di agiotoponomastica di M. R. Cerasuolo Pertusi, in corso di stampa), insenatura e porto più a $\mathrm{S}$. di Rabac, separata da questo da Porto Lungo e da altre località rivierasche minori.

3 In tempi più vicini a noi (secondo dopoguerra) Porto Albona risulta ulteriormente raccorciato in Port'Albona (raro, es. "Voce Giuliana" 16-1-1985) e Portalbona. 
ficato nuovo di rabac è nato per calco linguistico sulla voce venez. servidor, dal momento che rabac, in origine, significava "cattivo servo, servo ozioso" e sim. Semmai, per giustificare il passaggio di cotesto rabac a toponimo sarà necessario supporre che in epoca medievale, nella baia di Rabac, esisteva una salina, e questo secondo un'usanza di tempi remoti, quando qualsiasi specchio d'acqua poco profondo e in vicinanza della costa, data la necessità di procurarsi ad ogni costo il preziosissimo sale, veniva sistemato, magari attraverso tecniche rudimentali, a salina (cfr. Zamboni cit. p. 266). E il fiumiciattolo chiamato Rabac che sfocia nel porto omonimo potrebbe aver fatto parte, originariamente, dell'annesso sistema di canalizzazioni. Dall'idronimo, poi, a denominazione del porto e di tutta la baia circostante il passo è breve. Naturalmente bisogna partire, comeasi è già accennato, per rabac dal significato originario "cattivo servitore" (forse preso in senso benevolo, scherzoso) e rammentare che rabac è un peggiorativo di ant. sl. eccl. rab "servitore" (cfr. anche il verbo cr. ragus. rabačati "non far nulla, oziare"; su tutto ciò ci informa adeguatamente P. Skok ERHSJ III, s. v. rabačati).

\section{ABBREVIAZIONI IMPIEGATE}

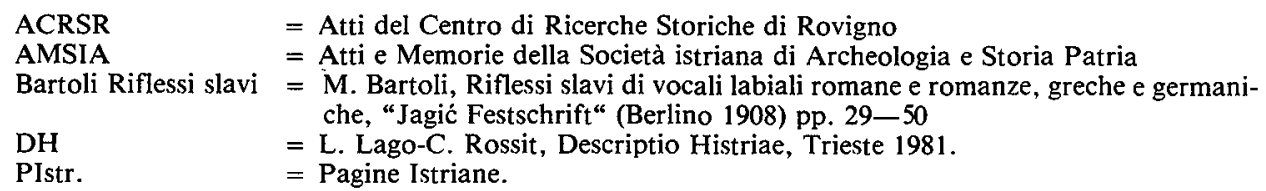

Povzetek

K ZGODOVINI ISTRSKEGA KRAJEVNEGA IMENA $R A B A C$

Rabac se kot krajevno ime pojavlja že v srednjeveških labinskih statutih. Splošno sprejeta etimologija ga ima za izpeljanko iz imena otoka $R a b$. Avtor vendar meni, da bi za trdnost take razlage morali poznati ime naselja, ki naj bi ga bilo novo poimenovanje nadomestilo. Predlaga neko drugo možno razlago: Rabac je tudi ime hudournika, ki se izliva $\mathrm{v}$ istoimensko pristanišč; tako bi rabac lahko bil izraz za odvodni kanal v solinah, za kar je v Istri običajen izraz servidor. Pomenski premik nikakor ni presenetljiv: $r a b$ je v cerkveni slovanščini '(božji) služabnik', prim. SKOK, ERHSJ III, s. v. rabàcati. Rabac bi bil potemtakem pomenski kalk po istrskobeneškem servidor. 